MODELING, IDENTIFICATION AND CONTROL, 1983, VOL. 4, NO. 2, 101-105

doi: $10.4173 /$ mic.1983.24

\title{
A war of area attrition and aimed attack-properties of optimal strategies
}

\author{
K. M. MJELDE†
}

Keywords: defence analysis, differential games.

\begin{abstract}
A war is considered of the attrition of the enemy's weapons supply system or the attack of his forces in the battle area. The rate of weapons supply of each side depends on the total number of successful attrition allocations of the other side; this dependency is described by a decreasing and differentiable function. The attack allocations are aimed at specific targets, while the attrition allocations are randomly distributed over the area in which the supply operations take place. The theory of differential games is applied to derive properties of optimal solutions.
\end{abstract}

\section{Introduction}

A differential game theoretic model of a war of prescribed duration is considered, each side allocates weapons to the attrition of the enemy's weapons supply system or to the attack of his forces in the battle field; the objective of the attack allocations is to change the rate of movement of the front line or to inflict casualties. Such models have been considered by Isaacs (1965), Berkovitz and Dresher (1960, 1957), Fulkerson and Johnson (1957) and Mjelde (1980). Several authors have analysed the problem in the context of the determination of an optimal distribution of supporting fire (artillery) between the enemy's primary forces (infantry) and his supporting forces. Taylor (1978) develops results for a Lanchester-type differential game; some other works are mentioned: Bellman and Dreyfus (1958); Giamboni, Mengel and Dishington (1951), Kawara (1973) and Weiss (1959). Mjelde (1982) considered the problem of the defence of a valuable target against enemy attacks over a certain period of time. until reinforcements arrive; using control theoretical arguments.

Mjelde (1980) introduced a rate of supply of (supporting) weapons of each side that decreased when the number of successful attrition allocations of the other side increased. The attrition and attack allocations were assumed to correspond to aimed fire, with an effectiveness proportional to the rate of fire. In this paper the attrition fire is randomly distributed over the area where the supply activities take place; the number of successful attrition allocations of each side increases at a rate proportional to the product of the rates of weapons supply of each side. The attack allocations, however, are aimed at selected targets.

It is demonstrated that an optimal solution concentrates all fire either to attrition or to attack, and that there is at most one transition from attrition to attack allocations; the war ends with all fire applied to attack. A condition is given for each side to start the battle with attrition allocations.

Received 23 November $\mathbf{9} 982$.

† Det norske Veritas, Veritasveien 1, 1322 Høvik, Norway. 


\section{The model}

The model considered in this paper is denoted by $P$ and defined as follows:

$$
P: V=\min _{\phi_{1}} \max _{\phi_{2}} \int_{0}^{T}\left[r_{2} g_{2}\left(x_{2}\right)\left(1-\phi_{2}\right)-r_{1} g_{1}\left(x_{1}\right)\left(1-\phi_{1}\right)\right] d t
$$

subject to

$$
\begin{gathered}
\dot{x}_{1}=\gamma_{1} g_{1}\left(x_{1}\right) g_{2}\left(x_{2}\right) \phi_{2} \\
\dot{x}_{2}=\gamma_{2} g_{1}\left(x_{1}\right) g_{2}\left(x_{2}\right) \phi_{1} \\
0 \leqslant \phi_{i} \leqslant 1 \text { for } i=1,2 \\
x_{i}(0)=0 \text { for } i=1,2
\end{gathered}
$$

The $x_{i}(t)$ and $\phi_{i}(t)$ for $i=1,2$ are functions of the time $t \geqslant 0$ and $\dot{x}_{i}=d x_{i} \mid d t$. The $\gamma_{t}$ and the $r_{t}$ for $i=1,2$ are positive real constants and the $g_{t}$ for $i=1,2$ are continuously differentiable functions with $g_{i}\left(x_{i}\right)>0$ and $g_{i}^{\prime}\left(x_{i}\right)=d g_{i}\left(x_{i}\right) \mid d x_{i}<0$ for all $x_{i} \geqslant 0$.

Defining

$$
i^{*}=\left\{\begin{array}{l}
1 \text { if } i=2 \\
2 \text { if } i=1
\end{array}\right.
$$

the quantities of the model are given as follows:

$x_{i}(t)$ : the total number of attrition allocations inflicted on side $i$ by side $i^{*}$ in the time interval $[0, t]$.

$g_{i}\left(x_{i}\right)$ : the rate of weapons supply of side $i$ as a function of $x_{i}(t)$.

$\phi_{i}(t)$ : the fraction of $g_{t}\left(x_{i}\right)$ allocated by side $i$ to attrition at time $t$; the remaining weapons are allocated to attack.

$\gamma_{i}$ : the effectiveness of an attrition allocation by side $i^{*}$.

$r_{i}$ : the effectiveness of an attack allocation by side $i$.

$T$ : the duration of the war.

\section{Optimality conditions}

If $V_{i}$ denotes the partial derivative of $V$ with respect to $x_{i}$ for $i=1,2$, the main equation of differential game theory, see Isaacs (1965) and Friedman (1971), shows that optimal solution(s) satisfy:

$$
\min _{\phi_{1}} \max _{\phi_{2}}\left\{-C_{3}+r_{2} g_{2}\left(x_{2}\right)-r_{1} g_{1}\left(x_{1}\right)+\phi_{1} g_{1}\left(x_{1}\right) S_{1}+\phi_{2} g_{2}\left(x_{2}\right) S_{2}\right\}=0
$$

for some constant $C_{3}$ and

$$
\begin{aligned}
& S_{1}=V_{2} \gamma_{2} g_{2}\left(x_{2}\right)+r_{1} \\
& S_{2}=V_{1} \gamma_{1} g_{1}\left(x_{1}\right)-r_{2}
\end{aligned}
$$

Any optimal solution of $P$ satisfy:

$$
\phi_{1}=\left\{\begin{array}{ll}
0 & \text { if } S_{1} \geqslant 0 \\
1 & \text { if } S_{1}<0
\end{array} ; \quad \phi_{2}= \begin{cases}0 & \text { if } S_{2} \leqslant 0 \\
1 & \text { if } S_{2}>0\end{cases}\right.
$$


If

$$
\tau=T-t
$$

denotes the retrogressive time and

$$
\check{V}_{i}=d V_{i} \mid d \tau \text { and } \check{S}_{i}=d S_{i} \mid d \tau
$$

the path equations are given by:

$$
\begin{aligned}
& \check{V}_{1}=-r_{1} g^{\prime}{ }_{1}\left(x_{1}\right)+\phi_{1} g^{\prime}{ }_{1}\left(x_{1}\right) S_{1}+\phi_{2} g^{\prime}{ }_{1}\left(x_{1}\right) g_{2}\left(x_{2}\right) V_{1} \gamma_{1} \\
& \stackrel{\circ}{2}_{2}=r_{2} g^{\prime}{ }_{2}\left(x_{2}\right)+\phi_{2} g_{2}^{\prime}\left(x_{2}\right) S_{2}+\phi_{1} g_{1}\left(x_{1}\right) g^{\prime}{ }_{2}\left(x_{2}\right) V_{2} \gamma_{2}
\end{aligned}
$$

with the initial conditions:

$$
V_{1}=V_{2}=0 \text { for } \tau=0
$$

The differentiation of the $S_{i}$ given by eqns. (3) and (4) with respect to $\tau$ gives:

$$
\begin{aligned}
& \stackrel{\Omega}{S}_{1}=\gamma_{2} g_{2}\left(x_{2}\right) g^{\prime}{ }_{2}\left(x_{2}\right)\left(r_{2}+\phi_{2} S_{2}\right)+V_{2} \gamma_{2} g^{\prime}{ }_{2}\left(x_{2}\right)\left[\gamma_{2} g_{1}\left(x_{1}\right) g_{2}\left(x_{2}\right) \phi_{1}+\dot{x}_{2}\right] \\
& \stackrel{8}{S}_{2}=\gamma_{1} g_{1}\left(x_{1}\right) g^{\prime}{ }_{1}\left(x_{1}\right)\left(-r_{1}+\phi_{1} S_{1}\right)+V_{1} \gamma_{1} g^{\prime}{ }_{1}\left(x_{1}\right)\left[\gamma_{1} g_{1}\left(x_{1}\right) g_{2}\left(x_{2}\right) \phi_{2}+\dot{x}_{1}\right]
\end{aligned}
$$

where the expressions inside the square brackets are both equal to zero, because of eqns. (1) and (2) and the relationship $\dot{x}_{1}=-\dot{x}_{i}$ (which follows from $\tau=T-t$ ). The implication is that:

$$
\begin{aligned}
& \varsigma_{1}=\gamma_{2} g_{2}\left(x_{2}\right) g^{\prime}{ }_{2}\left(x_{2}\right)\left[r_{2}+\phi_{2} S_{2}\right] \\
& \S_{2}=\gamma_{1} g_{1}\left(x_{1}\right) g^{\prime}{ }_{1}\left(x_{1}\right)\left[-r_{1}+\phi_{1} S_{1}\right]
\end{aligned}
$$

with the initial conditions:

$$
S_{1}=r_{1} \text { and } S_{2}=-r_{2} \text { for } \tau=0
$$

Since $\phi_{2} S_{2} \geqslant 0$ and $\phi_{1} S_{1} \leqslant 0$ the following result is valid:

Lemma 1

$$
S_{1}(\tau)<0, \quad S_{2}(\tau)>0 \quad \text { for } \tau \in[0, T]
$$

\section{Properties of optimal solutions}

An optimal solution of $P$ is denoted by $\phi_{i}^{*}(t)$ for $i=1,2$.

Equations (5) and (10) show that $\phi_{1}{ }^{*}=\phi_{2}{ }^{*}=0$ for $\tau=0$.

Integration of eqns. (8) and (9) with the initial condition (10) and $\phi_{1}{ }^{*}=\phi_{2}{ }^{*}=0$ demands that $\phi_{1}{ }^{*}$ or $\phi_{2}{ }^{*}$ changes from 0 to 1 at times $\tau_{1}\left(s_{2}\right)$ or $\tau_{2}\left(s_{1}\right)$, where

$$
\begin{aligned}
& \tau_{1}\left(s_{2}\right)=-r_{1} / r_{2} \gamma_{2} g^{\prime}{ }_{2}\left(s_{2}\right) g_{2}\left(s_{2}\right) \\
& \tau_{2}\left(s_{1}\right)--r_{2} / r_{1} \gamma_{1} g^{\prime}{ }_{1}\left(s_{1}\right) g_{1}\left(s_{1}\right)
\end{aligned}
$$

and

Define:

$$
s_{i}=x_{i}(T) \text { for } i=1,2
$$

$$
\begin{array}{ll}
\tau_{10}=\operatorname{Min} \tau_{1}\left(s_{2}\right) & \text { for } 0 \leqslant s_{2} \leqslant \gamma_{2} g_{1}(0) g_{2}(0) T \\
\tau_{20}=\operatorname{Min} \tau_{2}\left(s_{1}\right) & \text { for } 0 \leqslant s_{1} \leqslant \gamma_{1} g_{1}(0) g_{2}(0) T
\end{array}
$$

Lemma 1 and the previously given observations give: 


\section{Theorem I}

Any optimal solution has the following properties:

(1) $\phi_{1} *=\phi_{2} *=0$ for $\tau \leqslant \operatorname{Min}\left(\tau_{10}, \tau_{20}\right)$

(2) $\phi_{i}{ }^{*} \in\{0,1\}$ for $i=1,2$ and $\phi_{i}^{*}(t)$ changes from 1 to 0 at most once (in forward time).

Figure 1 illustrates the simultaneous development of $\left(S_{1}, S_{2}\right)$ and the associated changes of $\left(\phi_{1}, \phi_{2}\right)$.

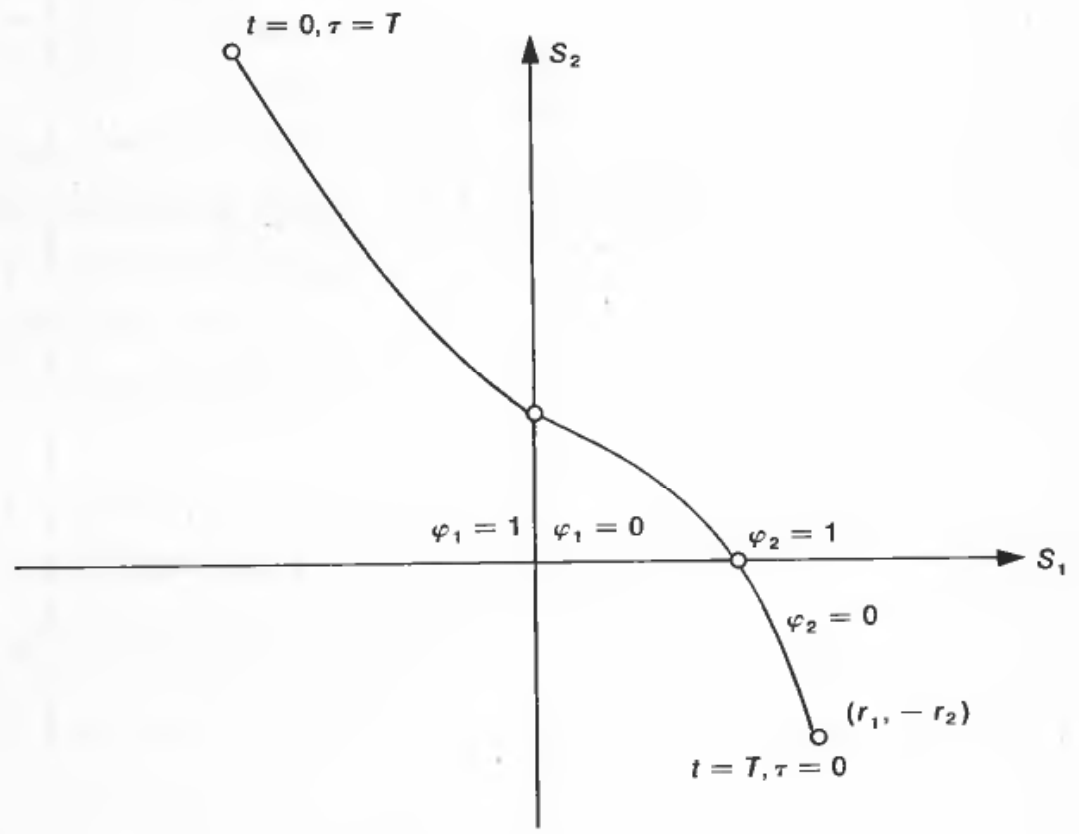

Figure 1. The $\left(S_{1}, S_{2}\right)$ curve.

\section{Theorem 2}

If $T>\tau_{i}(0)$ then $\phi_{i} *(0)=1 \quad$ for $i=1,2$.

\section{Proof}

Consider the case $i=1$ and assume, by contradiction, that $\phi_{1} *(0)=0$. Theorem 1 demands that $\phi_{1}{ }^{*}(t)=0$ for $t \in[0, T]$ and eqn. (1) that $x_{2}{ }^{*}(t)=0$. Since $\left(r_{2}+\phi_{2}{ }^{*} S_{2}{ }^{*}\right)>$ $r_{2}$ eqn. (8) requires that $S_{1} *(t) \leqslant \bar{S}_{1}(t)$ where $\bar{S}_{1}(t)$ is given by:

$$
\stackrel{\stackrel{\partial}{S}}{S_{1}}=\gamma_{2} g_{2}(0) g_{2}^{\prime}(0) r_{2}
$$

and

$$
\bar{S}_{1}(0)=r_{1}
$$

Since $\bar{S}_{1}(\tau)=0$ for $\tau=\tau_{1}(0)$ and $T>\tau_{1}(0)$ it follows that $\phi_{1}{ }^{*}(0)=1$. q.e.d. 


\section{Final remarks}

Let $x_{i}{ }^{*}$ and $S_{i}{ }^{*}$ denote the values of $x_{i}$ and $S_{i}$ corresponding to optimal allocations $\phi_{i}=\phi_{i}{ }^{*}$ for $i=1,2$. Any given lower and upper bounds of $\phi_{i}^{*}(t)$ for $t \in[0, T]$ and $i=1,2$ (for instance defined by $0 \leqslant \phi_{i}^{*}(t) \leqslant 1$ ), can be used to derive bounds of $x_{i}{ }^{*}(t)$ for $t \in(0, T]$ and $i=1,2$ by the application of equations and arguments analogous to those of Mjelde (1982). The bounds of $\phi_{i}{ }^{*}$ and $x_{i}{ }^{*}$ can be used to derive bounds of $S_{i}{ }^{*}$, and corresponding new bounds of $\phi_{i}{ }^{*}$, and this process can be continued. These bounds are useful in the solution of the problem $P$ by numerical methods that require initial estimates of the quantities $\phi_{i}{ }^{*}, x_{i}{ }^{*}$ or $S_{i}{ }^{*}$, see for instance Bryson and Ho (1969).

Problems for future work are the analysis of models with several types of weapons on each side. Other extensions are given for the introduction of a criterion that terminates the war, for instance if side 1 wins for $V=\underline{V}$ and side 2 wins for $V=\bar{V}$, where $\underline{V}<\bar{V}$.

\section{REFERENCES}

Bellman, R., and Dreyfus, S. (1958). On a tactical air-warfare model of Mengel. Operations Research, 6, 65-78.

Berkovitz, L., and Dresher, M. (1960). Allocation of two types of aircraft in tactical air war: a game theoretic analysis. Operations Research, 8, 694-706.

Berkovitz, L., and Dresher, M. (1959). A game-theory analysis of a tactical air war. Operations Research, 7, 594-620.

Bryson, E. A., and Ho, Y. C. (1969). Applied optimal control (Ginn and Company).

Friedman, A. (1971). Differential games. New York: Wiley.

Fulkerson, D., and Johnson, S. A tactical air game. Operations Research, 5, 704-712.

Giamboni, L., Mengel, A., and Dishington, R. (1951). Simplified model of a symmetric tactical air war. The RAND Corporation, RM-711.

IsAACS, R. (1965). Differential games. New York: Wiley.

KAWARA, Y. (1973). An allocation problem of fire support in combat as a differential game. Operations Research, 21, 942-951.

MJeLDE, K. M. (1982). The defence of a valuable target-a control theoretical analysis. Modeling, Identification and Control, 3, 1-10.

MJELDE, K. M. (1980). A war of attrition and attack with decreasing rates of weapons' supply. Cahiers du Centre d'Etudes de Recherche Operationelle (Bruxelles), 22, 111-123.

TAYLOR, J. G. (1978). Differential-game examination of optimal time-sequential fire-support strategies. Nav. Res. Log, Quart., 25, 323-355.

WFISs, H. (1959). Some differential games of tactical interest and the value of a supporting weapon system. Operations Research, 7, 180-196. 